\title{
Evaluation of Piloted Inputs for Onboard Frequency Response Estimation
}

\author{
Jared A. Grauer* \\ NASA Langley Research Center, Hampton, Virginia, 23681 \\ Borja Martos ${ }^{\dagger}$ \\ University of Tennessee Space Institute, Tullahoma, Tennessee, 37388
}

\begin{abstract}
Frequency response estimation results are presented using piloted inputs and a real-time estimation method recently developed for multisine inputs. A nonlinear simulation of the F-16 and a Piper Saratoga research aircraft were subjected to different piloted test inputs while the short period stabilator/elevator to pitch rate frequency response was estimated. Results show that the method can produce accurate results using wide-band piloted inputs instead of multisines. A new metric is introduced for evaluating which data points to include in the analysis and recommendations are provided for applying this method with piloted inputs.
\end{abstract}

\section{Nomenclature}

$\begin{array}{ll}\text { Roman } & \\ a_{k} & k^{\text {th }} \text { multisine amplitude [rad] } \\ G(j \omega) & \text { frequency response } \\ \Im\{.\} & \text { imaginary part } \\ j & \text { imaginary number, } \sqrt{-1} \\ K & \text { multisine frequency set } \\ N & \text { number of data samples } \\ q & \text { pitch rate [rad/s] } \\ \Re\{.\} & \text { real part } \\ T & \text { data record length }[\mathrm{s}] \\ t & \text { time }[\mathrm{s}] \\ u & \text { input variable } \\ y & \text { output variable } \\ \|\cdot\| & \text { magnitude }[\mathrm{dB}] \\ L . & \text { phase angle }[\mathrm{deg}]\end{array}$

$\begin{array}{ll}\text { Greek } & \\ \gamma & \text { normalized power }\left[\mathrm{rad}^{2} / \mathrm{s}\right] \\ \Delta & \text { perturbation } \\ \delta & \text { control surface deflection [rad] } \\ \sigma & \text { standard deviation } \\ \phi_{k} & k^{t h} \text { multisine phase }[\mathrm{rad}] \\ \omega & \text { frequency [rad/s] } \\ \omega_{n} & \text { modal natural frequency }[\mathrm{rad} / \mathrm{s}]\end{array}$

Superscripts

Fourier transform

Subscripts

e elevator

$s \quad$ stabilator

*Research Engineer, Dynamic Systems and Control Branch, MS 308, Member AIAA

${ }^{\dagger}$ Research Professor, Aviation Systems and Flight Research, MS 20, Member AIAA 


\section{Introduction}

Aircraft frequency responses are commonly estimated from measurements of input/output flight data. This is an attractive technique for modeling new or exotic aircraft because no prior knowledge about the dynamics is required. Furthermore, frequency response estimation can determine closed-loop models and stability margins for aircraft with new control laws, or can provide linear approximations for complex and nonlinear systems including pilot models and adaptive control laws. Traditionally, a frequency sweep is conducted on a control surface once the aircraft is on condition. After the flight data is downloaded, software routines ${ }^{1,2,3}$ apply Welch's method to compute frequency responses and coherence. ${ }^{1,2,3,4}$ Because this method requires a significant amount of time and money, ${ }^{3,5}$ recent studies have expedited data transfer using wireless or onboard hardware, ${ }^{6,7,8,9,10}$ or have used more efficient inputs. ${ }^{2,11,12,13,14}$

A method was recently developed for estimating frequency responses in real time. ${ }^{15,16}$ Orthogonal phaseoptimized multisine excitation inputs ${ }^{17,2,12,18}$ were used to excite all control surfaces simultaneously using a wide-band input at a discrete number of frequencies. A recursive Fourier transform ${ }^{19,2,12}$ was employed to update input and output frequency domain data at those discrete frequencies. Afterwards, frequency responses were computed, from which amplitude, phase, and stability margins were extracted. Noise covariances were propagated through this process to estimate the accuracy of this information. Simulation and flight test data showed accurate short period frequency responses were obtained within 5-10 s. Results also showed that sudden changes in the dynamics, for example due to structural damage, were resolved in the estimates within $9 \mathrm{~s}$ using a sliding window of data. This algorithm can be employed to shorten wind tunnel and free flight test durations, assist reconfigurable control laws, monitor the aircraft health, and monitor stability margins for new or adaptive aircraft.

This study investigated the degree to which the frequency response method developed for multisine inputs performs using piloted inputs. This is important and relevant because in many cases it is prohibitively expensive or difficult to retrofit and certify aircraft with hardware and instrumentation to use multisine excitation inputs. A nonlinear simulation of the F-16 aircraft and a Piper Saratoga research aircraft were subjected to several different piloted inputs and their frequency responses were estimated between the stabilator/elevator input and the pitch rate output in the region of the short period mode. Results showed that the method can be used with piloted inputs to compute estimates that are within statistical agreement of truth solutions obtained using either numerical linearization or output-error parameter estimation. A metric was proposed for evaluating which frequencies to include in the analysis, and recommendations were suggested for implementing this method with piloted inputs.

The remainder of this paper is organized as follows. The F-16 nonlinear simulation and Piper Saratoga are described in Section II. Section III summarizes the the piloted types, the multisine inputs, and the frequency response estimation method. Section IV presents the results for the simulation and flight test data. Section V discusses lessons learned and provides recommendations for applying the frequency response method using piloted inputs.

Computer programs to design the inputs, perform output-error parameter estimation, and simulate the nonlinear F-16 aircraft are included in a MATLAB ${ }^{\circledR}$ toolbox called System IDentification Programs for AirCraft (SIDPAC). ${ }^{2}$ This software was developed at NASA Langley Research Center, and is continually expanded and improved upon.

\section{Materials}

Two sources were used to obtain data for this study. The first source was a nonlinear simulation of the rigid-body flight dynamics of the General Dynamics F-16, which is shown in Fig. 1(a). This aircraft is a single-seat jet fighter with a nominal 637 slug mass, $32.7 \mathrm{ft}$ wing span, and $300 \mathrm{ft}^{2}$ reference wing area. Control inputs include the throttle setting and the stabilator, aileron, and rudder control surface deflections. The aerodynamic model consists of lookup tables from static and forced oscillation wind tunnel tests using a $16 \%$ scale test article. ${ }^{20}$ Lookup tables were modified by removing the second-order effects of the longitudinal motion on the sideslip angle. ${ }^{21}$ Engine dynamics include the gyroscopic effects of the spinning turbo machinery and a first-order lag, identified from ground tests, between the throttle command and the thrust output. Output measurements were corrupted using white Gaussian random noise sequences.

The F-16 nonlinear simulation is included as MATLAB ${ }^{\circledR}$ code in SIDPAC, as well as additional software for trimming the aircraft and generating linear perturbation models using central finite differences. For the 


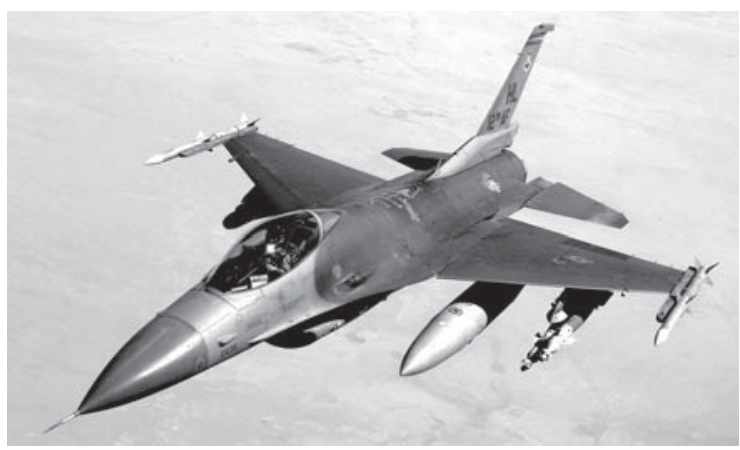

(a) F-16 (credit: US Defense Imagery)

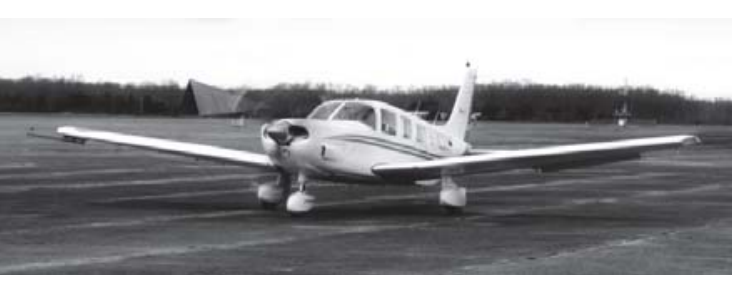

(b) UTSI Piper Saratoga

Figure 1. Test aircraft

results presented in this paper, the F-16 simulation was trimmed for straight and level flight at 10,000 ft with an angle of attack of $4.0 \mathrm{deg}$ and an airspeed of $490 \mathrm{ft} / \mathrm{s}(0.45 \mathrm{Mach})$. Numerical linearization reported the short period mode at $2.17 \mathrm{rad} / \mathrm{s}$. The stabilator deflection and pitch rate measurement noise standard deviations were $0.11 \mathrm{deg}$ and $0.39 \mathrm{deg} / \mathrm{s}$, respectively, which are similar to the Piper Saratoga. Simulation data were sampled at $50 \mathrm{~Hz}$.

The second source of data was the Piper Saratoga, PA-32-301, tail number N22UT, shown in Figure 1(b). This aircraft is owned and operated by the University of Tennessee Space Institute (UTSI). The stock version is a high performance, fixed-wing aircraft that can seat two pilots and four flight test engineers. The aircraft has fixed landing gear, a maximum take-off weight of $3600 \mathrm{lbs}$, a $32 \mathrm{ft}$ wingspan, and a $174.5 \mathrm{ft}^{2}$ wing area. It is equipped with a single $300 \mathrm{hp}$ Lycoming IO-540-K1G5 engine that drives a constant-speed, three-bladed Hartzell propeller. The maximum speed of the aircraft is $253 \mathrm{ft} / \mathrm{s}$, due to structural limits, and the stall speed is $105 \mathrm{ft} / \mathrm{s}$ in the clean configuration.

The stock aircraft comes equipped with a pitot-static tube. The aircraft has been retrofitted with an additional temperature probe, Kiel probe, total pressure port, and a static pressure probe. An inertial navigation system was also installed to provide acceleration, rotational velocity, inertial velocity, and attitude measurements. Control surfaces have been instrumented with load cells and potentiometers for measuring loads and deflections. An air data boom has also been installed for measuring the angle of attack and sideslip angle. Data were recorded at $20 \mathrm{~Hz}$ using an onboard data acquisition system. For flight data presented in this study, the aircraft was trimmed for straight and level flight at $6400 \mathrm{ft}$ with a $4.0 \mathrm{deg}$ angle of attack and a $180 \mathrm{ft} / \mathrm{s}$ airspeed $(0.16 \mathrm{Mach})$.

\section{Methods}

\section{A. Conventional Test Inputs}

This section summarizes the conventional pilot test inputs, which consist of impulses, doublets, multistep inputs, and frequency sweeps. Other references ${ }^{22,23,2,3,18}$ contain a complete description and guidelines for designing these inputs.

The most basic and easiest to implement of the conventional pilot test inputs is the impulse, which consists of a sudden spike to the control surfaces. Impulses have the advantage in that they are wide-band inputs, but also the disadvantage that they contain little spectral power.

A second input is the doublet. For this input, a control surface is perturbed in one direction for a specified amount of time, then perturbed in the opposite direction for the same amount of time, and then returned to the unperturbed position. The recommended time duration for each pulse width is $\pi / \omega_{n}$, which centers the input power spectrum around the expected natural frequency of interest, $\omega_{n}$. The amplitudes of the perturbations are selected to excite the dynamics above noise and turbulence levels and to keep the aircraft near the trimmed flight condition. These inputs are easy to implement and excite the transient response behavior, but do not contain much spectral power.

Another pilot input is the 3-2-1-1 multistep input, which is a series of alternating steps. Each step has 
time durations as multiples of a fundamental pulse width, hence the name. The fundamental pulse width is recommended as $0.7 \pi / \omega_{n}$ to center the power spectrum. This input has a broader frequency spectrum, but the long initial step can take the aircraft off the flight condition, and it does not have even energy in positive and negative perturbations. A good compromise between the doublet input and the 3-2-1-1 multistep is the 2-1-1 multistep. Although the energy is still uneven between the positive and negative input perturbations, this input has more power than a doublet input and is less likely to take the aircraft off the flight condition than the $3-2-1-1$. The recommended fundamental time period for this input is $\pi / 2 \omega_{n}$. All of these multistep inputs can be repeated for more excitation power or to balance the input energy.

A fourth pilot input is the frequency sweep. A sinusoidal input is applied, beginning at a low frequency and gradually increasing in frequency. Because a range of frequencies are excited, exact knowledge of modal frequencies of interest are not needed prior to testing. Amplitudes are often small in the beginning of the test to limit deviations from the flight condition while the low frequency inputs are applied. Additionally, if the sweep is performed slowly enough, transient responses are only minimally excited, which is good for modeling frequency responses. While frequency sweeps cover a broad spectral range, they often require relatively long records of data and have limited spectral power at any given frequency.

\section{B. Orthogonal Phase-Optimized Multisines}

This section describes the orthogonal phase-optimized multisine inputs, which were developed previously ${ }^{17,2,12,18}$ and are briefly summarized here. These perturbation inputs are added to all control surface deflection commands at the actuators and before deflection and rate limiters. Each input consists of the superposition of sinusoids

$$
u(t)=\sum_{k \in K} a_{k} \sin \left(\frac{2 \pi k}{T} t+\phi_{k}\right)
$$

where $K$ is the set of indices, $a_{k}$ is the amplitude, $T$ is the excitation record length, $\phi_{k}$ is the phase angle, and $\omega_{k}=2 \pi k / T$ is the excitation frequency. The first step in designing these inputs is to choose the excitation record length, which determines the fundamental frequency of the input. The available set of frequencies are then integer multiples of this fundamental frequency. The subset $K$ is chosen so that frequencies lay within a band of interest and can be assigned to control surfaces in any fashion and with arbitrary amplitude. Phase angles are then determined using a simplex optimization method to minimize input peak-to-peak amplitudes, which are scaled to specified values. For control-oriented modeling of conventional fixed-wing aircraft, uniform power is usually distributed in an alternating manner to control surfaces between $0.1 \mathrm{~Hz}$ and $2.0 \mathrm{~Hz}$. Peak-to-peak amplitudes are typically between 0.5 and 3.0 degrees, based on aircraft size, control power, dynamic response, and atmospheric turbulence.

These inputs are well-suited for identifying dynamic models in a time efficient manner, and have yielded good results in a variety of flight conditions including straight and level, hypersonic, high sideslip, and poststall flight. ${ }^{18,14}$ Because sinusoids are multiples of a fundamental frequency, all excitation frequencies can be applied simultaneously to multiple control surfaces without correlating the data. This fact dramatically reduces test durations because multiple inputs and frequencies no longer need to be excited sequentially. Additionally, multisine inputs encourage the emergence of the steady-state response, which is used in frequency response estimation. Harmonic amplitudes and peak-to-peak amplitudes are selected to target specific frequency bands and to excite the system above noise and turbulence levels. The optimized phase angles minimize the excursions from the trim condition, which is ideal for linear modeling about specific conditions.

\section{Frequency Response Estimation}

This section presents the frequency response estimation method developed by Grauer and Morelli, ${ }^{15,16}$ which uses input and output measurements to estimate the corresponding frequency response and uncertainty as a Bode plot. Measurements of the inputs and outputs are first transformed into the frequency domain. The finite Fourier transform, for example using the input measurement,

$$
\tilde{u}(j \omega)=\int_{0}^{T} u(t)[\cos (\omega t)-j \sin (\omega t)] d t
$$


can be evaluated at discrete time steps and specific frequencies as

$$
\tilde{u}\left(j \omega_{k}\right) \simeq \sum_{i=0}^{N-1} u\left(t_{i}\right)\left[\cos \left(\omega_{k} t_{i}\right)-j \sin \left(\omega_{k} t_{i}\right)\right] \Delta t
$$

where $N$ is the number of data samples and $\Delta t$ is the sampling interval. Given Fourier transforms of an input and output, the associated frequency response is

$$
G\left(j \omega_{k}\right)=\frac{\tilde{y}\left(j \omega_{k}\right)}{\tilde{u}\left(j \omega_{k}\right)}
$$

from which the Bode magnitude and phase angle

$$
\begin{aligned}
\left\|G\left(j \omega_{k}\right)\right\| & =\sqrt{\Re\left\{G\left(j \omega_{k}\right)\right\}^{2}+\Im\left\{G\left(j \omega_{k}\right)\right\}^{2}} \\
\angle G\left(j \omega_{k}\right) & =\arctan \left(\frac{\Im\left\{G\left(j \omega_{k}\right)\right\}}{\Re\left\{G\left(j \omega_{k}\right)\right\}}\right)
\end{aligned}
$$

are extracted. The uncertainty of the frequency response magnitude and phase angle estimates are computed by propagating noise covariances of the measurements through this analysis. ${ }^{15,16}$

Equations (2) through (5) are formulated for batch analysis, after all the data is collected. A recursive formulation of the Fourier transform, ${ }^{19,2,12}$ for example using the input measurement,

$$
\tilde{u}_{i}\left(\omega_{k}\right)=\tilde{u}_{i-1}\left(\omega_{k}\right)+u\left(t_{i}\right)\left[\cos \left(\omega_{k} t_{i}\right)-j \sin \left(\omega_{k} t_{i}\right)\right] \Delta t
$$

updates the Fourier transform as each new data point is recorded, where $i=0,1, \ldots, N-1$ are the indices. Afterwards, frequency response estimates and their uncertainties can be updated at any desired rate. Data forgetting and windowing algorithms can be incorporated to account for systems with time-varying dynamics. Perturbations can be computed in real time from the measured flight data by either subtracting off recorded trim conditions or by high pass filtering the data. Good results have been obtained in the past using a fourth-order Butterworth filter with the break frequency below the lowest excitation frequency. ${ }^{15,16,12}$

The frequency response estimation algorithm was originally intended to use the multisine inputs to excite the aircraft. In this case, the excitation time $T$ and the excitation frequencies $\omega_{k}$ are already known since they were designed before the maneuver. The Fourier transforms were evaluated at the excitation frequencies, so that only a few points in the frequency domain were used and these points had excellent signal-to-noise ratios since they contained the excitation. When the orthogonal phase-optimized multisines are not employed and instead pilot inputs are used, the input energy does not occur at discrete peaks in the frequency spectrum and engineering judgment is needed to choose where the Fourier transform is evaluated. One choice is to use the finest spacing possible in the frequency domain, $1 / T$, but this requires knowledge of the maneuver duration. This fine spacing also leads to a large number of points to evaluate, e.g. when using a frequency sweep, which can be prohibitive during real-time estimation. A useful choice is to arbitrarily select the spacing as $0.1 \mathrm{~Hz}$, which represents a good trade-off between resolution on the frequency response and computation time. However it is possible that some of these arbitrarily chosen points do not contain much excitation and lead to inaccurate estimates of the frequency response. A measurement of the normalized input power can be used to determine which frequencies are used in the analysis. The normalized input power

$$
\gamma_{i}\left(j \omega_{k}\right)=\gamma_{i-1}\left(j \omega_{k}\right)+\frac{1}{N \Delta t}\left\|\tilde{u}_{i}\left(j \omega_{k}\right)\right\|_{2}^{2}
$$

can be evaluated recursively. Once this value is above a specified threshold value, the frequency $\omega_{k}$ is incorporated into the analysis and produces accurate results. Simulation cases and flight data presented in Section IV indicated that $20 \mathrm{deg}^{2} / \mathrm{s}$ was a good threshold value for stabilator or elevator excitation. Another simpler possibility would be to discard estimated frequency response points that have relatively large error bounds.

\section{Results}

This section presents the frequency response estimation results. In each case, the pitch dynamics of the aircraft were excited near the short period mode and the frequency response from the stabilator or elevator to the pitch rate was examined. Although any input/output pair could be used, this combination was selected for analysis because the second-order short period mode dynamics are well known. 


\section{A. Simulation Batch Results}

The F-16 nonlinear flight dynamics simulation was used to test the frequency response estimation to different inputs. In this section, results are presented using the entire $20 \mathrm{~s}$ data record, which includes $16 \mathrm{~s}$ of excitation. Inputs with less duration were repeated. The amplitude of the inputs were tailored so that the aircraft did not exceed control surface deflection limits or perturbations larger than $5 \mathrm{deg}$ angle of attack, $10 \mathrm{deg} / \mathrm{s}$ pitch rate, and $1 \mathrm{~g}$ heave acceleration. Inputs were recorded using a desktop simulation. All frequency responses were estimated between $0.1 \mathrm{~Hz}$ and $2.0 \mathrm{~Hz}$, in $0.1 \mathrm{~Hz}$ increments. This range encapsulates the short period dynamics and represents a good trade-off between computation time and resolution on the frequency response. This spacing requires $10 \mathrm{~s}$ of data. If fewer points are used, detail in the estimate is lost. If more points are used, more computation time and longer data records are needed.

A multisine was first applied to the stabilator to establish a baseline case for comparison with the piloted inputs. Harmonic amplitudes were uniformly distributed and the resulting waveform was scaled for a $2.5 \mathrm{deg}$ peak-to-peak amplitude. Stabilator and pitch rate perturbations are shown in Figure 2(a). Figure 2(b) shows the frequency spectrum of the input and output. There are 20 sinusoids summed together in this input, hence 20 peaks in the input Fourier transform. Because this aircraft resembles a linear system for small perturbations about the flight condition, there are also 20 distinct peaks in the output Fourier transform. These 20 points have excellent signal-to-noise ratio and are the points at which the frequency response is estimated. Application of Equations 3 through 5 is shown in Figure 2(c), with $2 \sigma$ error bars and the solution obtained from numerical linearization using central finite differences. The error bounds are relatively small and the estimates are in statistical agreement with the finite difference result. Figure 2(d) shows the evolution of the normalized power for each one of the frequencies used in the frequency response estimate. It was found that $20 \mathrm{deg}^{2} / \mathrm{s}$ was a good threshold value to determine which points to use in the analysis. For the multisine input, all of the frequencies quickly pass through this threshold. This plot also indicates that good estimates of the frequency response can be made within $5-10 \mathrm{~s}$, which is consistent with previous results. ${ }^{15,16}$

Results using an impulse, having a 15 deg amplitude and repeating every $1 \mathrm{~s}$ with alternating signs, are shown in Figure 3. The amplitude was designed to be large enough that the response is above the noise floor and small enough to remain within the limits of the stabilator deflection range. Figure 3(b) shows that the input power is concentrated about two narrow peaks. As a result, only two frequencies crossed the threshold in Figure 3(d) and only a few estimates in Figure 3(c), near the resonant frequency, were both accurate and low in error.

The multistep doublet, $2-1-1$, and $3-2-1-1$ inputs are shown in Figures 4 through 6 . The "1" pulse width lengths were selected as $1.4 \mathrm{~s}, 1.0 \mathrm{~s}$, and $0.7 \mathrm{~s}$, respectively. As the frequency content of the input is varied, the peak amplitude in the input frequency spectrum diminishes, but the average amplitude increases, resulting in wide-band dynamic responses. As this happens, more frequency points used have richer frequency content and pass the $20 \mathrm{deg}^{2} / \mathrm{s}$ threshold, resulting in better frequency response estimates. The doublet only obtained good estimation near the resonant frequency, which may have been due to the higher signal-to-noise ratio that naturally occurs there. At low and high frequencies, there was little frequency content in the input which resulted in poor estimates with large error. The largest peak in the input frequency spectrum occurred at $1 \mathrm{rad} / \mathrm{s}$; however, because this was a sharp peak and because the Fourier transform was not evaluated near this peak, good dynamic information was discarded in the analysis. The $2-1-1$ and $3-2-1-1$ inputs have better low and middle frequency estimates because these inputs have broader frequency content. The peak amplitudes still occur near $1 \mathrm{rad} / \mathrm{s}$, but the other frequencies have large amplitudes as well. Both inputs however still lack excitation in the higher frequencies.

Figure 7 shows the results for using a frequency sweep. A full sinusoid with a $10 \mathrm{~s}$ period was applied, and then the frequency was increased for the remainder of the excitation. The input spectrum has a large peak at the low frequencies and then a relatively flat spectrum otherwise. It is recommended for piloted frequency sweeps ${ }^{3}$ that two cycles of the lowest frequency are applied, followed by a sweep that amounts to data records between 90-120 s, but this was changed to restrict the excitation time to $16 \mathrm{~s}$. Despite this change, most of the frequencies surpassed the $20 \mathrm{deg}^{2} / \mathrm{s}$ threshold and the frequency estimates were both accurate and low in error. The highest error estimates occurred at the highest frequencies, similar to the multistep inputs. However, this could be attenuated with more excitation. If a computer generated frequency sweep was used, the input spectrum in Figure 3(b) would be flat and the normalized power in Figure 3(d) would see sequential crossings of the threshold value as each frequency was excited. 

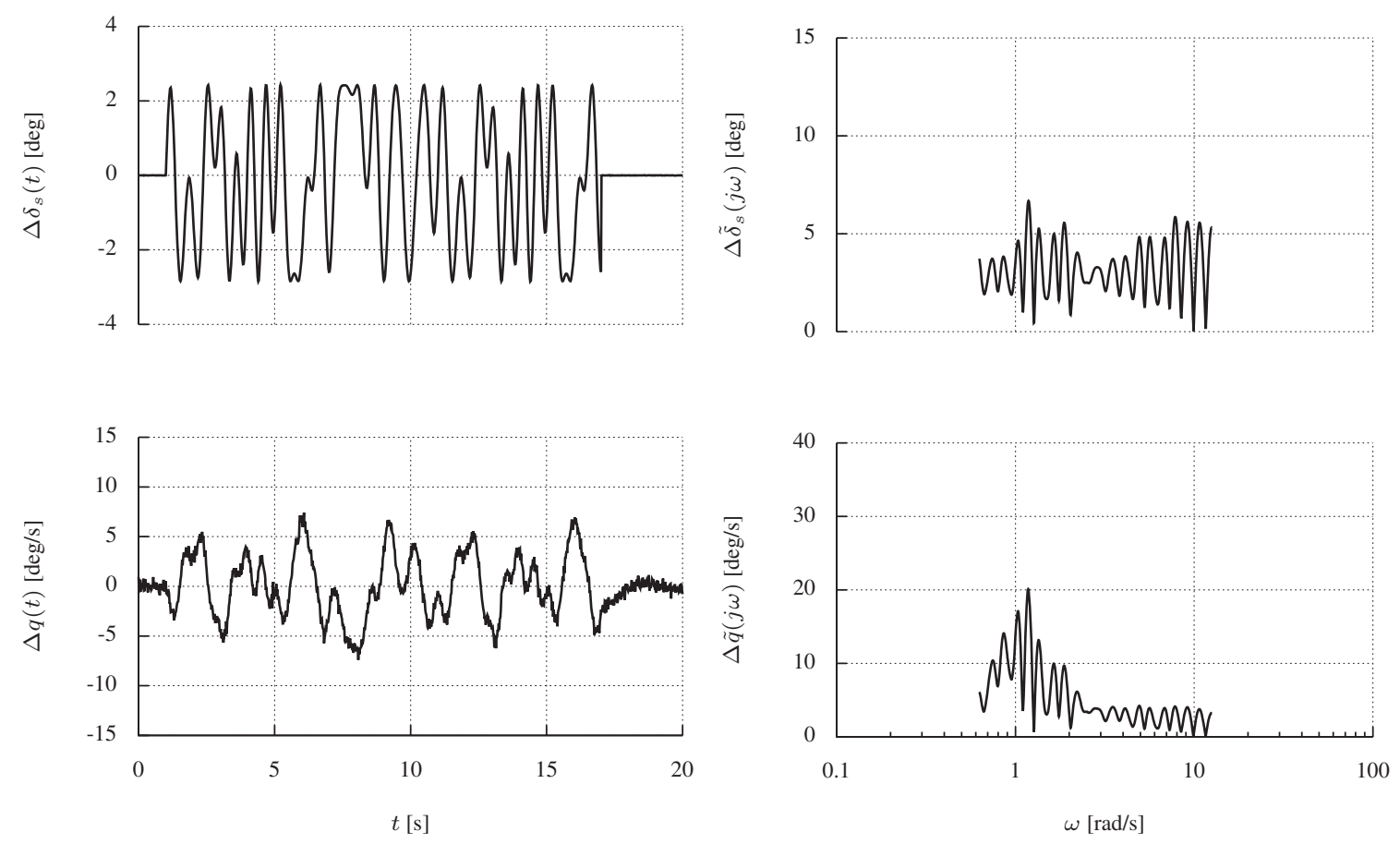

(a) time series data

(b) frequency spectrum

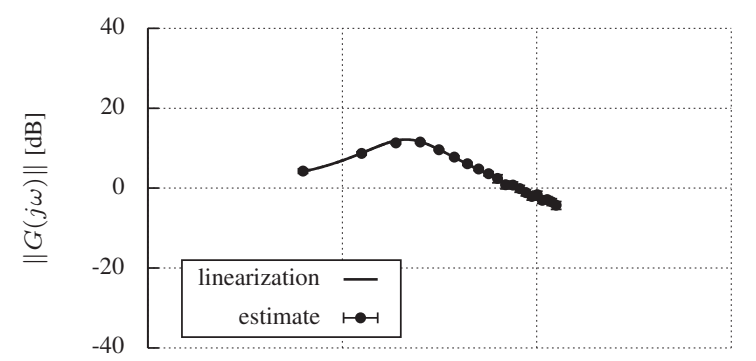

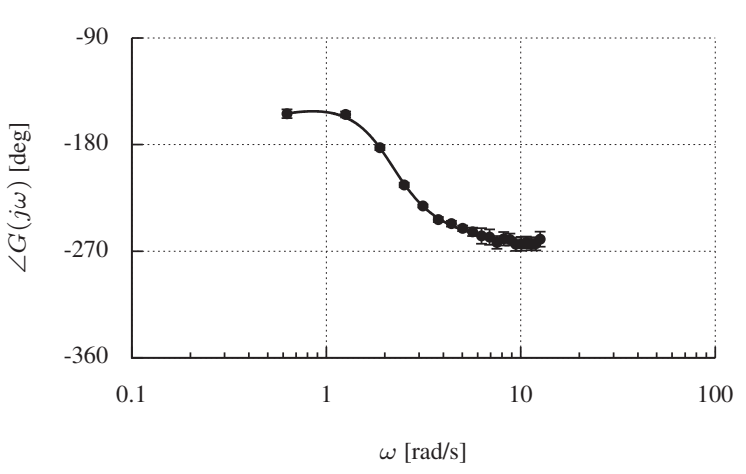

(c) frequency response

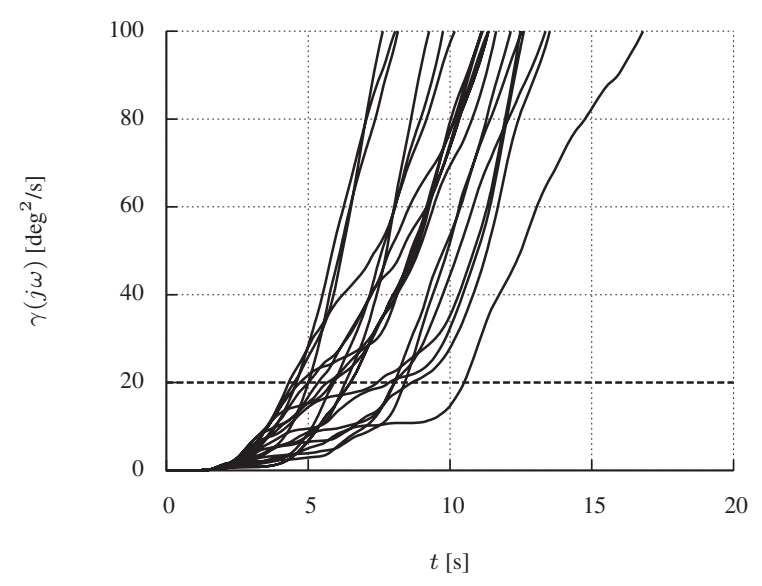

(d) normalized power

Figure 2. Simulation results using an orthogonal phase-optimized multisine input 

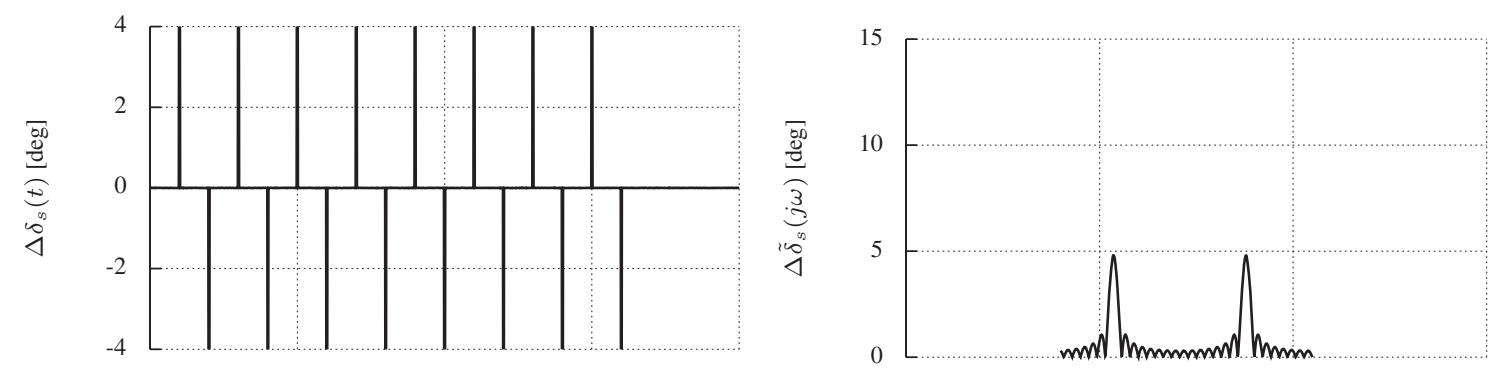

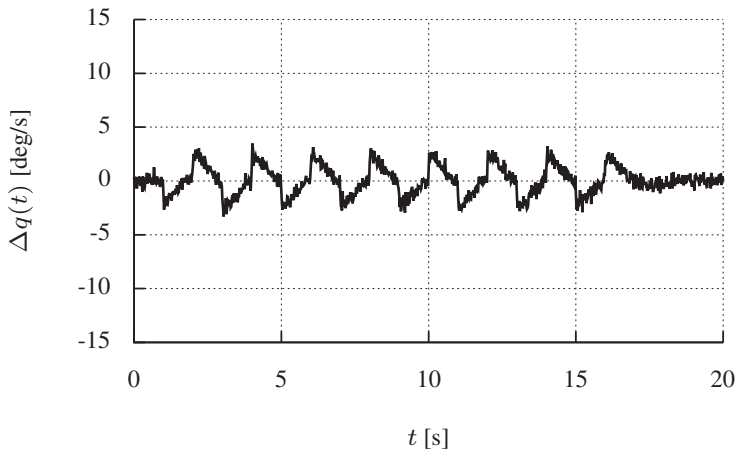

(a) time series data
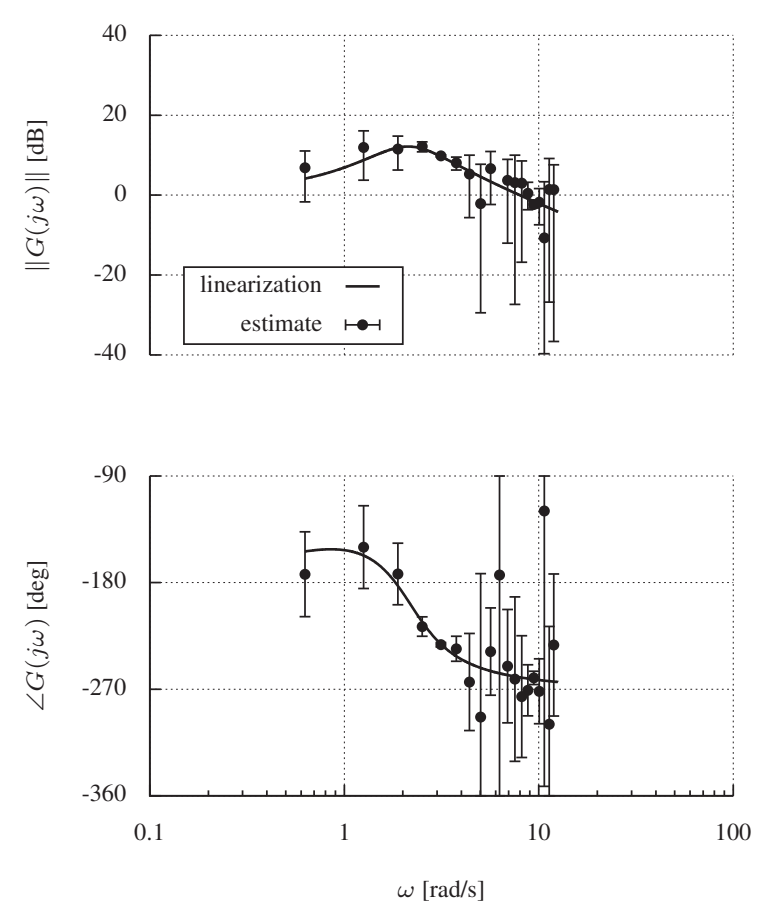

(c) frequency response

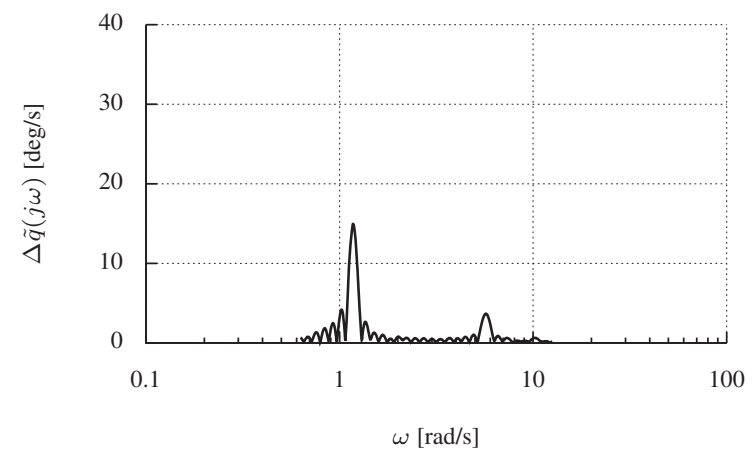

(b) frequency spectrum

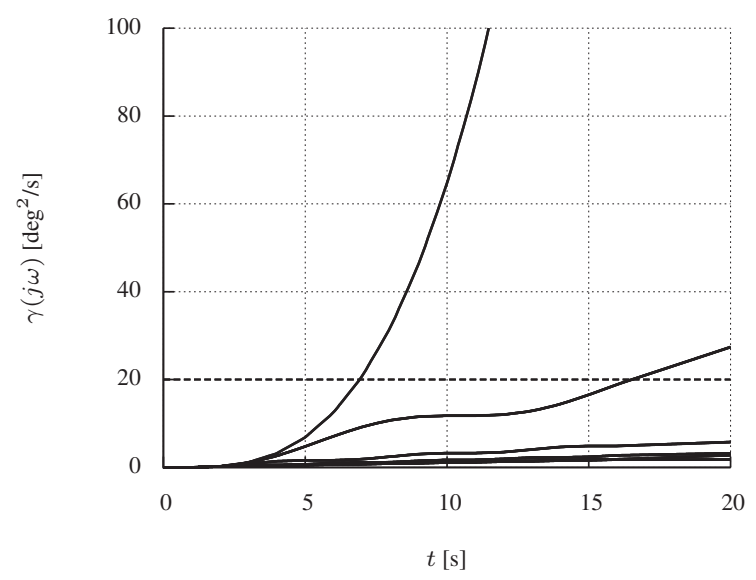

(d) normalized power

Figure 3. Simulation results using repeated impulses 

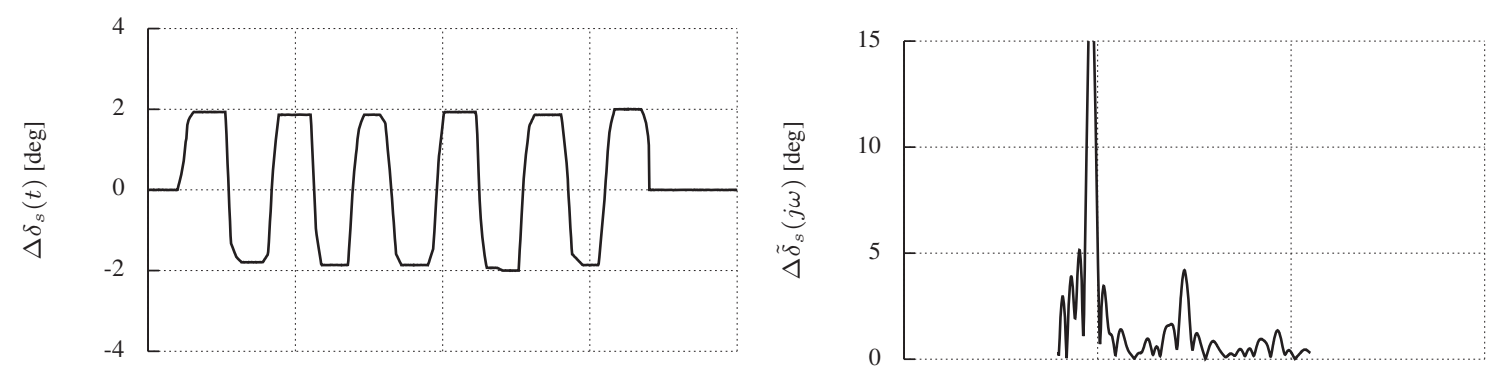

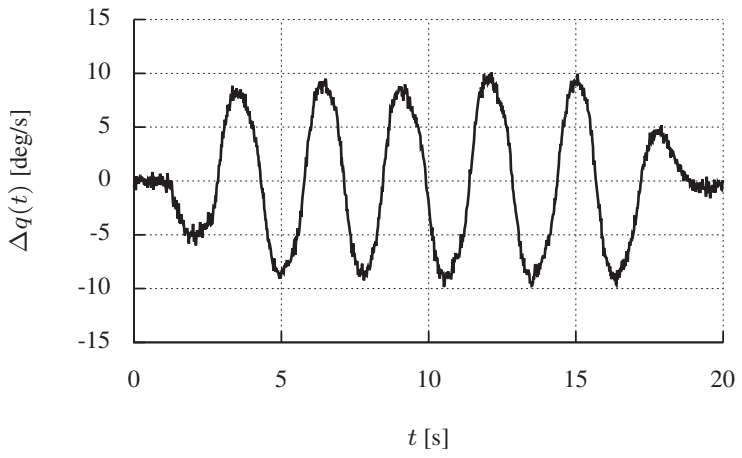

(a) time series data
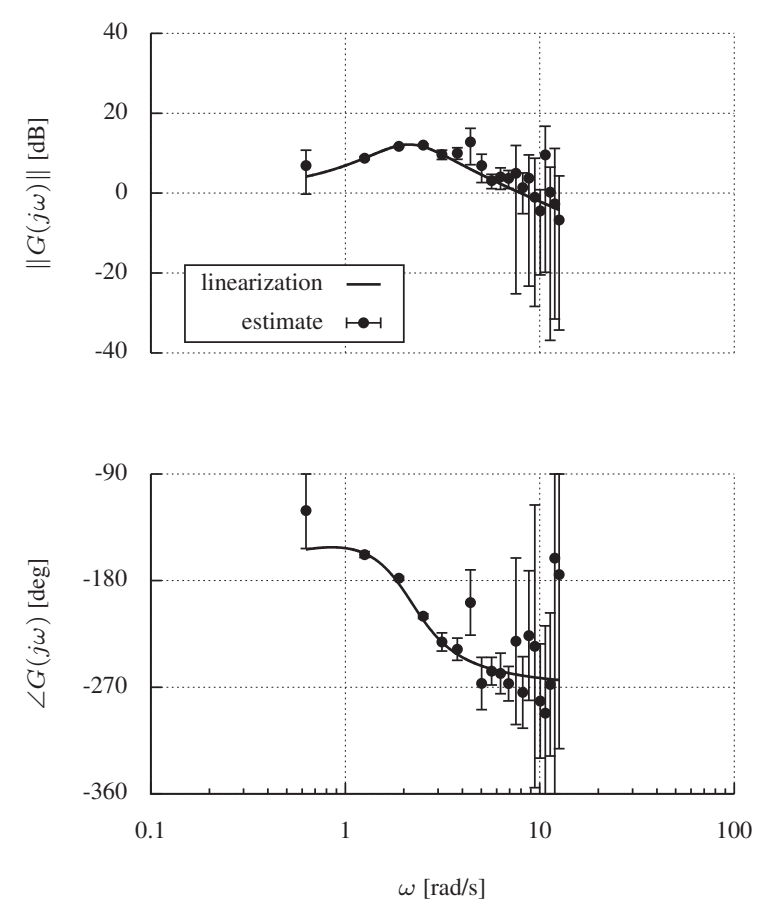

(c) frequency response

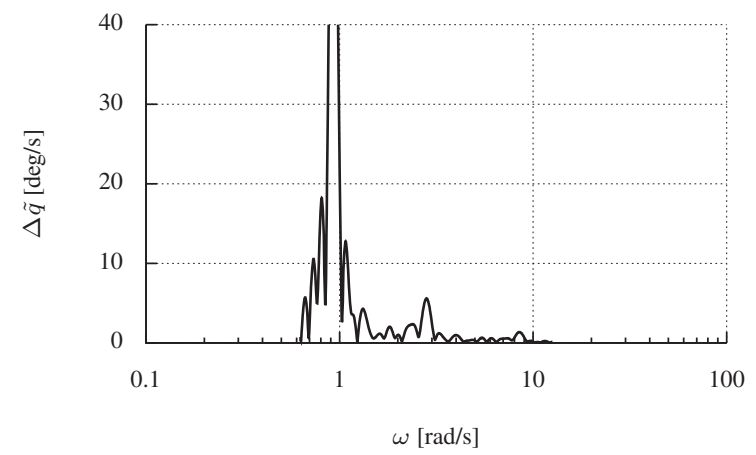

(b) frequency spectrum

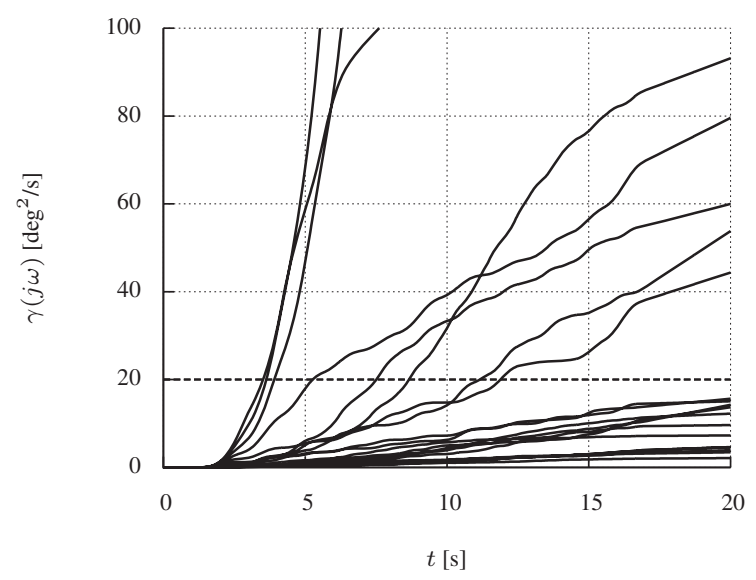

(d) normalized power

Figure 4. Simulation results using repeated doublets 

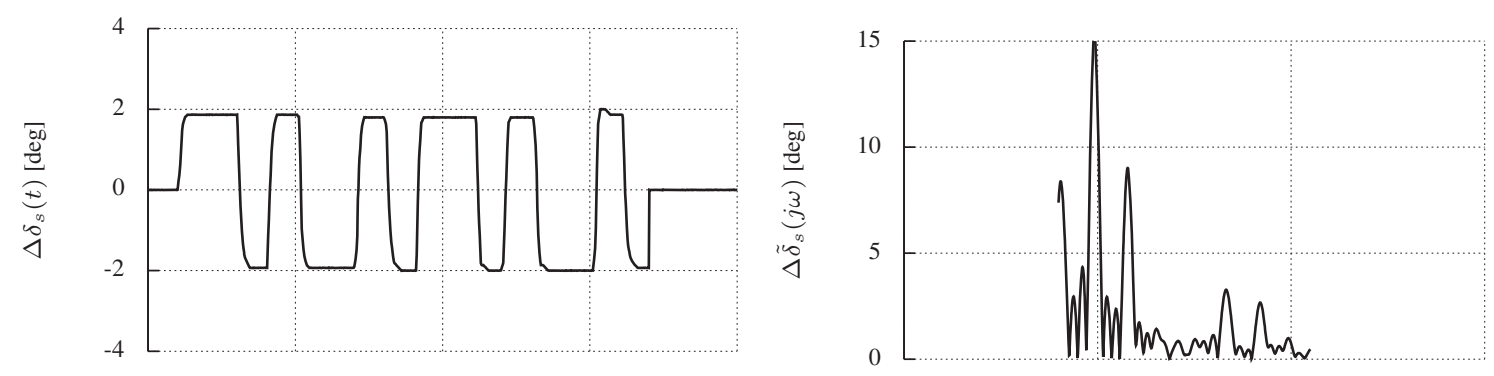

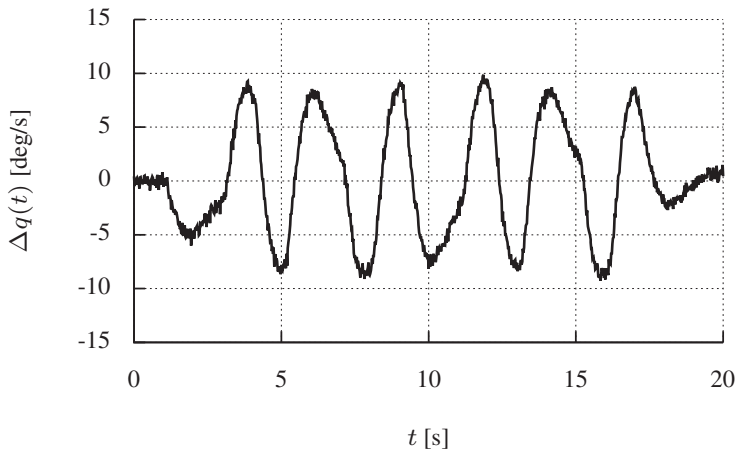

(a) time series data
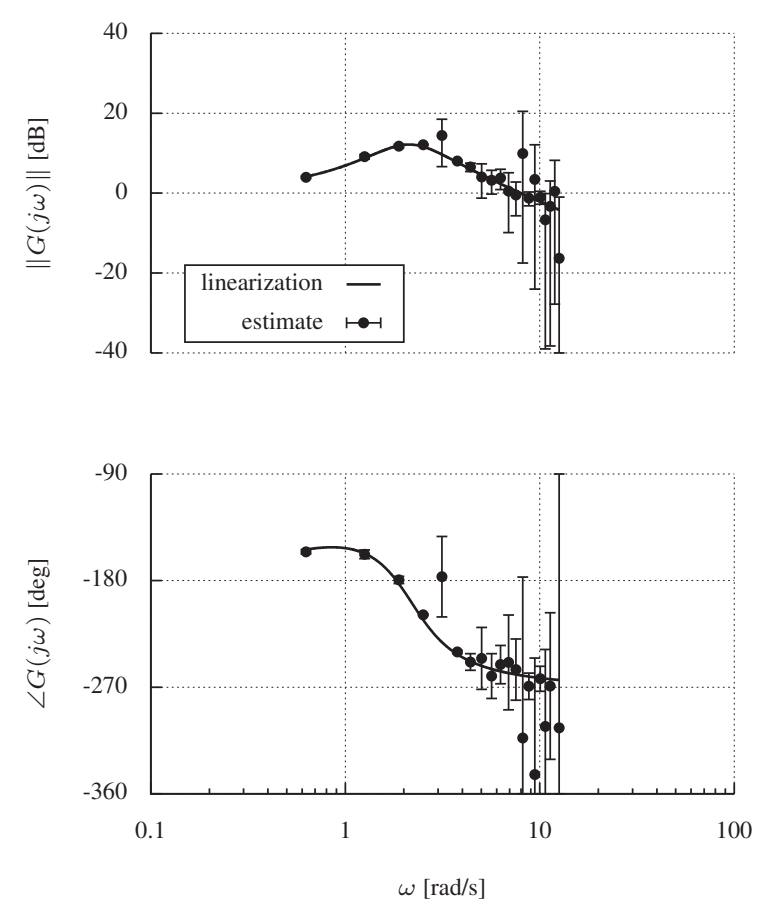

(c) frequency response

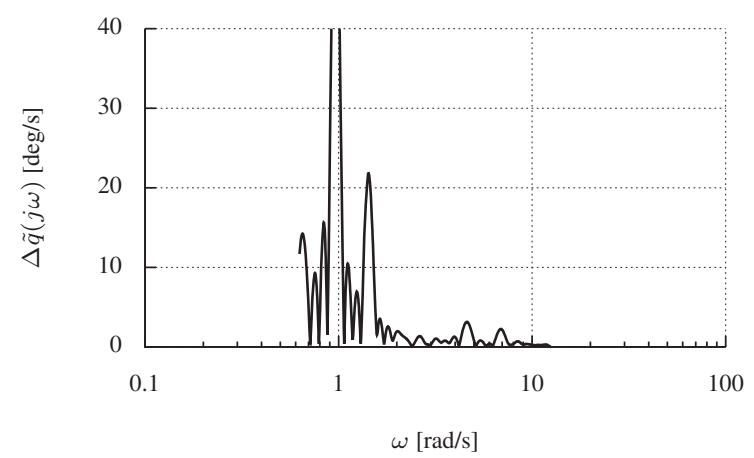

(b) frequency spectrum

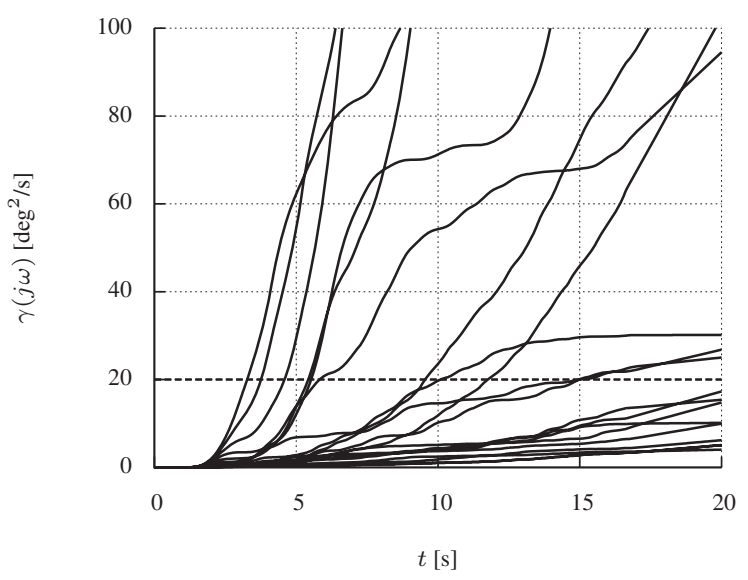

(d) normalized power

Figure 5. Simulation results using repeated 2-1-1 multisteps 

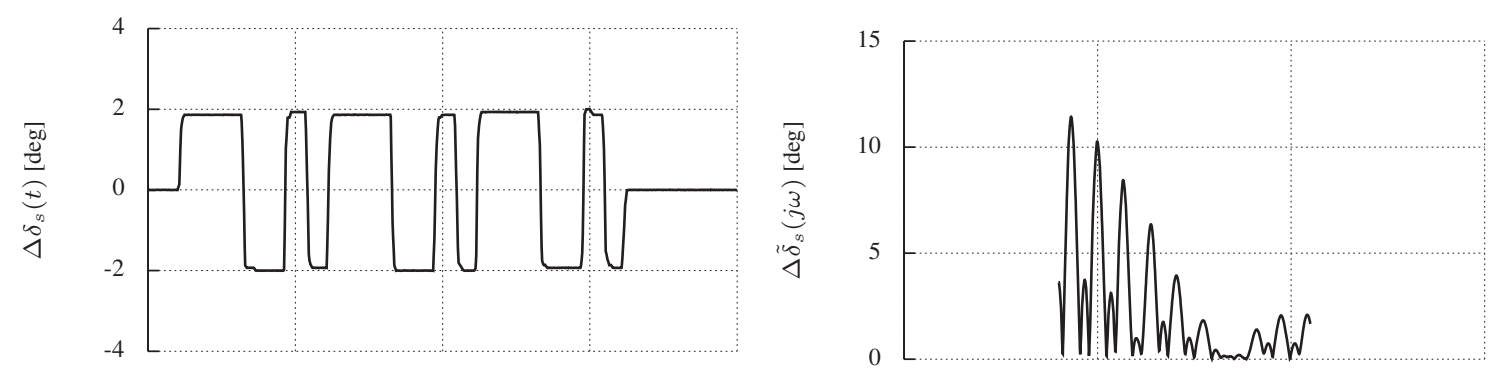

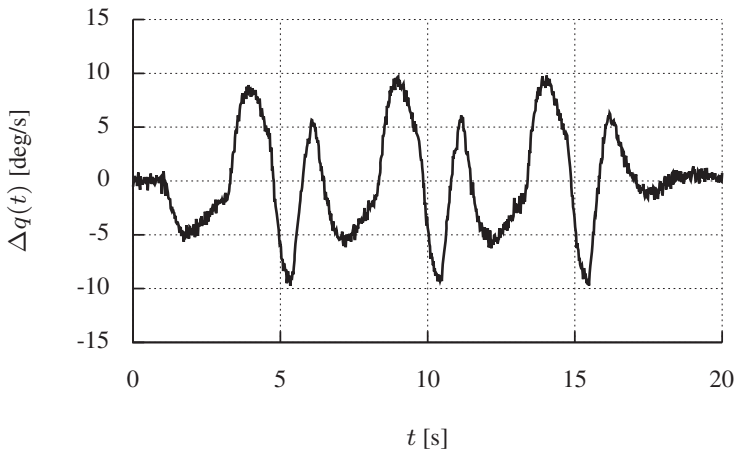

(a) time series data
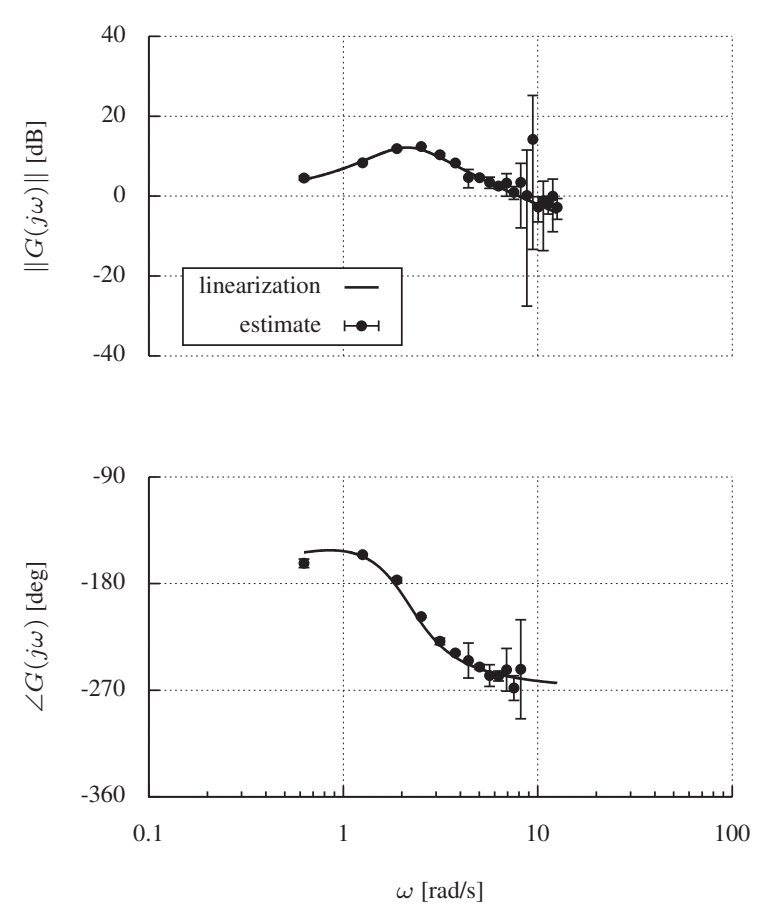

(c) frequency response

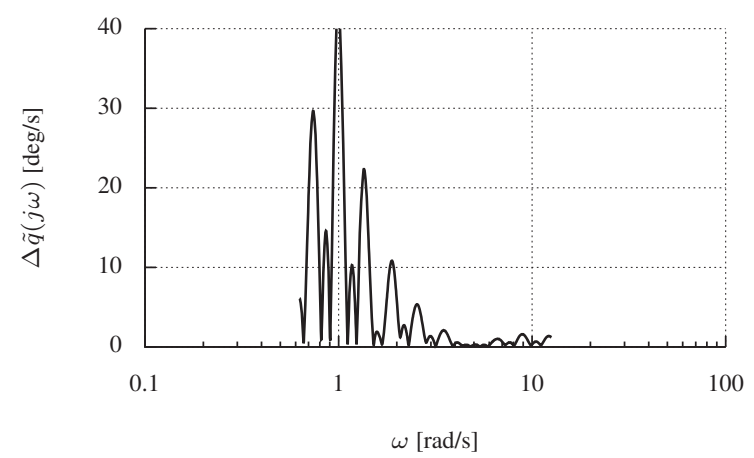

(b) frequency spectrum

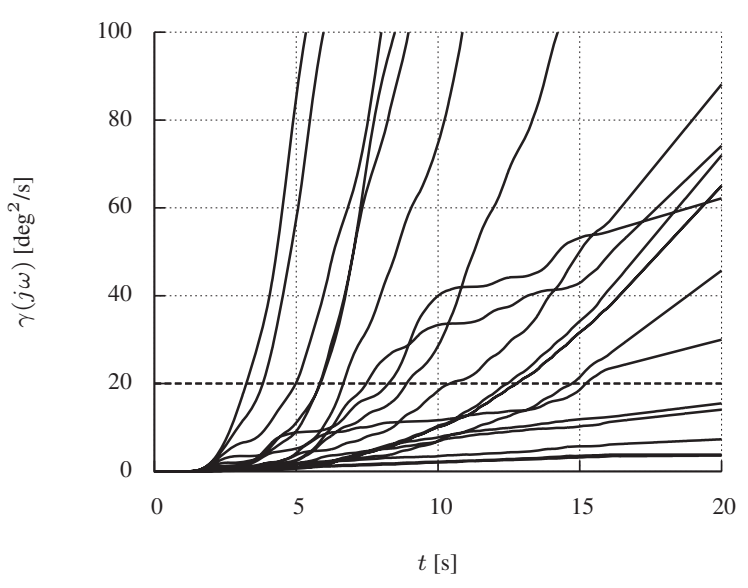

(d) normalized power

Figure 6. Simulation results using repeated 3-2-1-1 multisteps 

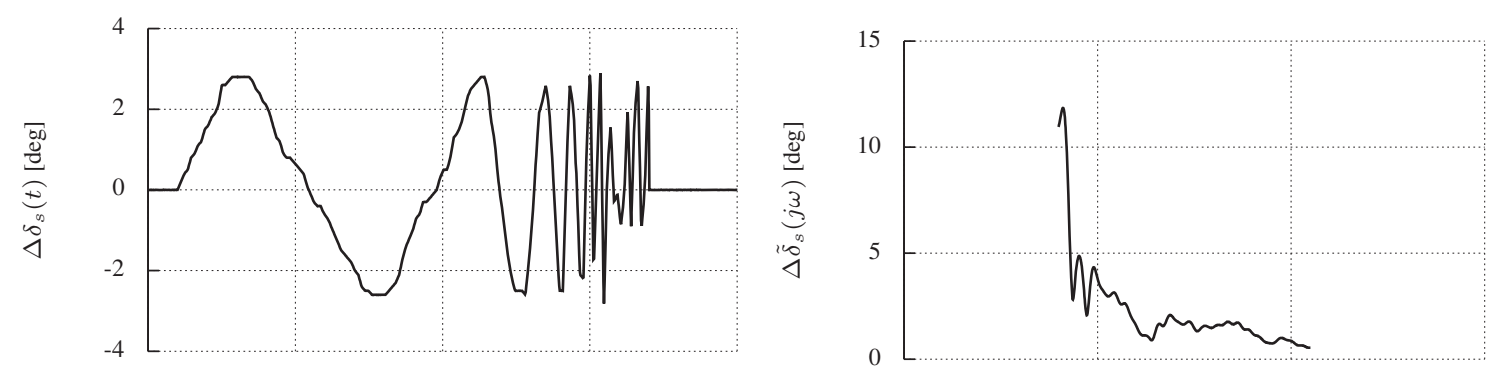

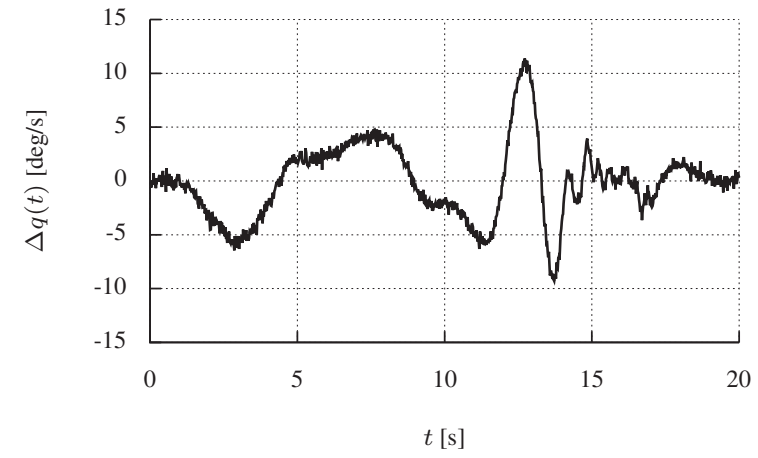

(a) time series data
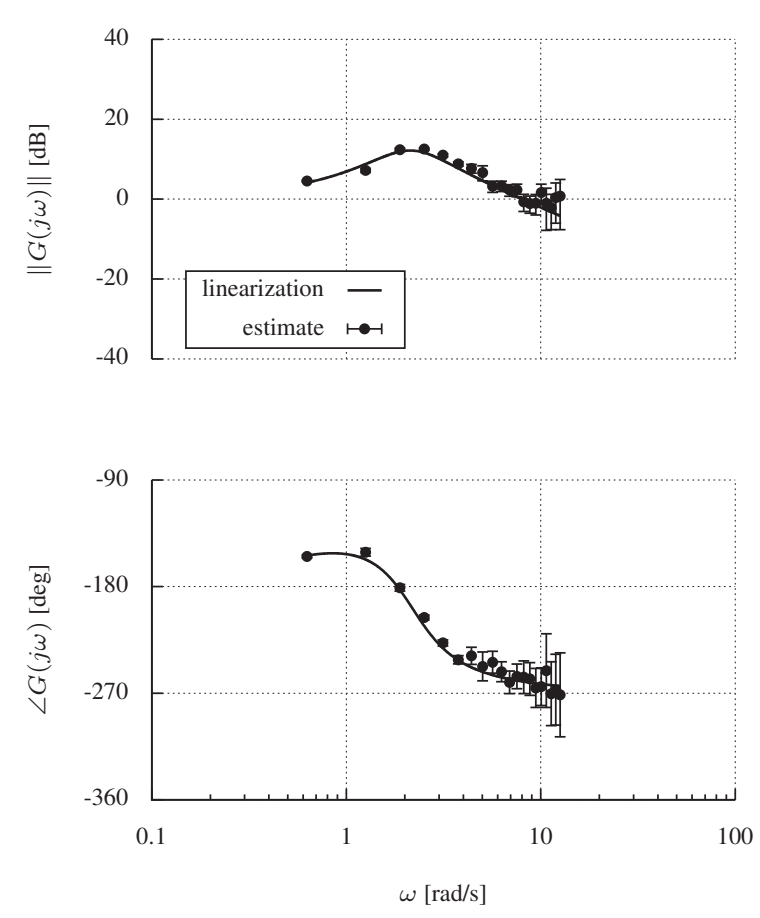

(c) frequency response

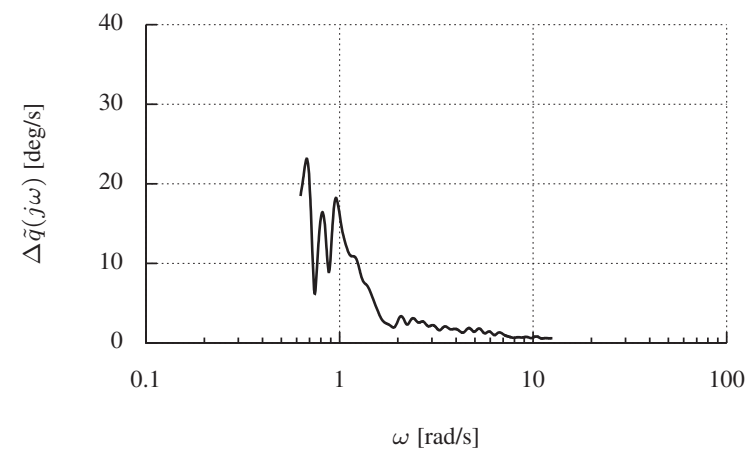

(b) frequency spectrum

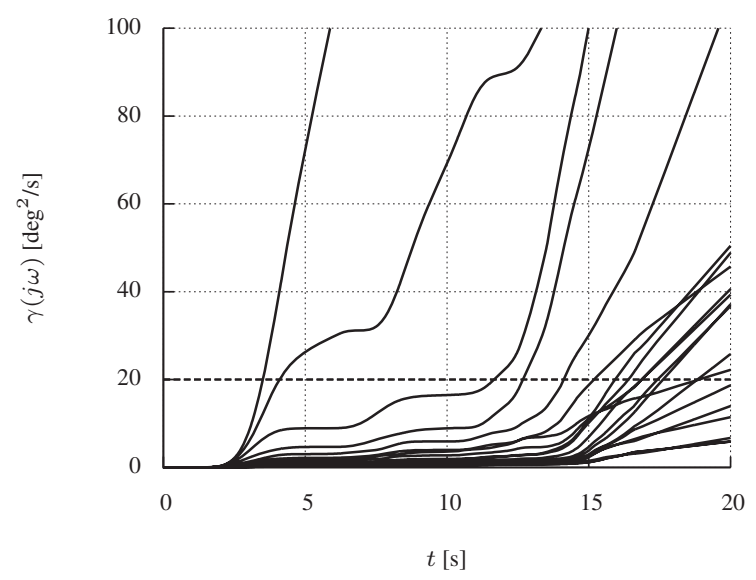

(d) normalized power

Figure 7. Simulation results using a frequency sweep 


\section{B. Flight Test Batch Results}

This section presents results using flight test data obtained from the Piper Saratoga onboard data logger. Only calibrations were applied to the measured signals; no filtering, smoothing, or kinematic consistency ${ }^{2}$ checking was applied to condition the data before analysis. No additional consideration was made for effects of turbulence or colored noise. The frequency grid for evaluation was selected as between $0.1 \mathrm{~Hz}$ and 2.0 $\mathrm{Hz}$, in $0.1 \mathrm{~Hz}$ increments. The frequency responses presented in this section were computing using the entire data record.

In the absence of a simulation model of this aircraft and numerical linearizations, the output-error method of parameter estimation ${ }^{24,2}$ was applied in the time domain using the flight test data to obtain a frequency response against which to compare the estimates. Output-error method, considered the industry standard, uses a model structure and an itereative nonlinear solver to estimate the parameters and their uncertainties in that model that best match the data to the model output. The pilot inputs and pitch rate outputs were used, as well as the angle of attack and heave acceleration measurements. The two-state short period mode approximation ${ }^{25,21,2}$ was used for the model structure. Initial estimates for the unknown stability and control derivatves were obtained using equation-error parameter estimation. ${ }^{2}$ The solver typically converged quickly within 18 iterations and coefficients of determination were above 0.96 for each measurement for each case, which indicated an accurate estimation. Once the parameters were identified, the corresponding frequency response was found as a truth model for comparison.

Figures 8 through 10 show results for a doublet input, a 3-2-1-1 multistep input, and a frequency sweep. Data records are different and there was no effort made to keep the excitation durations equal. Generally these results look similar to the simulation results. The doublet provides a similar result as the output-error at low frequencies, but then exhibits inaccuracies and large errors at the higher frequencies where there is low spectral content. The $3-2-1-1$ has a wider spread of power and is able to reduce some of the inaccuracies and errors at the higher frequencies. The frequency sweep used a longer duration of time, and likely because of that and wide-band excitation achieved good estimates throughout the spectrum of interest.

A non-conventional pilot input, nicknamed a "fuzzy" input, ${ }^{26}$ was also applied and is shown in Figure 11(a). The data record is approximately as along as the piloted frequency sweep. The idea of this input is to mimic a multisine input and excite the aircraft with a broad spectrum centered around the natural frequency of interest. The pilot adapts to the apparent motion of the aircraft and focuses more energy at the modal frequency. The frequency response estimation results are shown in Figure 11(b) and show the largest amount of inaccuracy and error at the lower frequencies. This is expected to be due to the excitation of the transient response of the aircraft, which is known to bias the low-frequency estimates. ${ }^{15,16}$ Inaccuracies are exhibited and large error bounds are seen in the higher frequency estimates.

\section{Real-Time Results}

The real-time version of the frequency response method was also applied to the simulation and flight test data. A baseline case was established using the F-16 nonlinear simulation and the multisine inputs shown in Figure 2(a), but repeated for $90 \mathrm{~s}$ to match frequency sweep and fuzzy input data records. Figures 12(a) and 12(b) show the evolution of the Bode magnitude and phase angle estimates over time, respectively. Also shown with the final estimates are the solutions computed using numerical linearization. Estimates vary at first but then converge within $10 \mathrm{~s}$ as more information becomes available to the algorithm. There is more oscillation at the higher frequencies because they have smaller signal-to-noise ratios.

The real-time method was also applied to the flight test data from the Piper Saratoga. Results using the frequency sweep and fuzzy input data in Figures 10(a) and 11(a) are shown in Figures 13 and 14, respectively. Final estimates are also plotted with results from an output-error analysis for comparison. While there is low frequency spectral content in the frequency sweep input, the lowest frequency used in the evaluation of the Fourier transform did not have much content, so that estimate is not very accurate. However as time progresses and the frequencies are passed, the frequency response estimate oscillates, evolves, and converges on the output-error estimate by the end of the maneuver. The fuzzy input has a different nature to its evolution. Frequencies oscillate and adapt as the pilot excites them with no particular order. Because the pilot was focused on exciting the short period mode of the aircraft, frequencies below and above that resonance are not as accurate. Unlike the frequency sweep where the lowest frequencies are deliberately excited, there is not much low frequency spectral content in the input. The frequency response estimates are however good near the short period mode of the aircraft. 

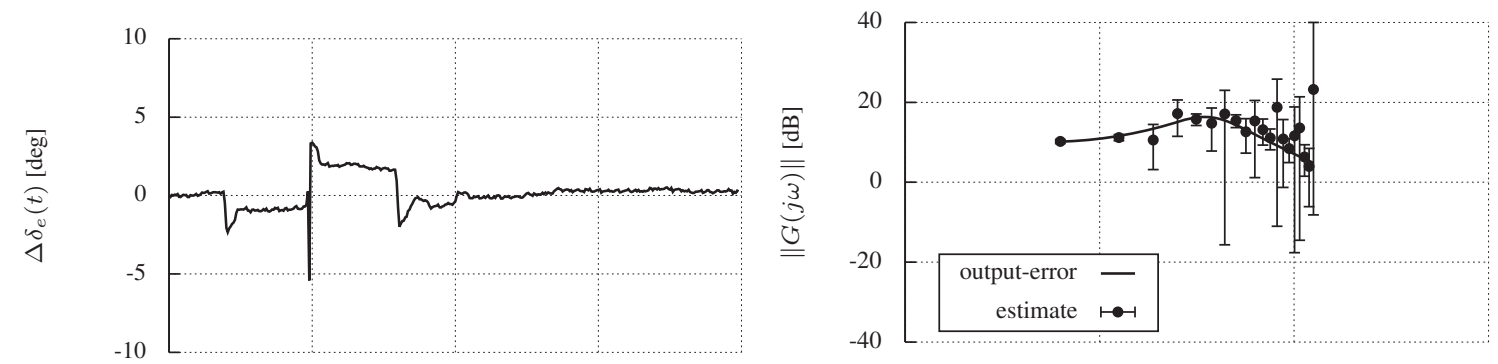

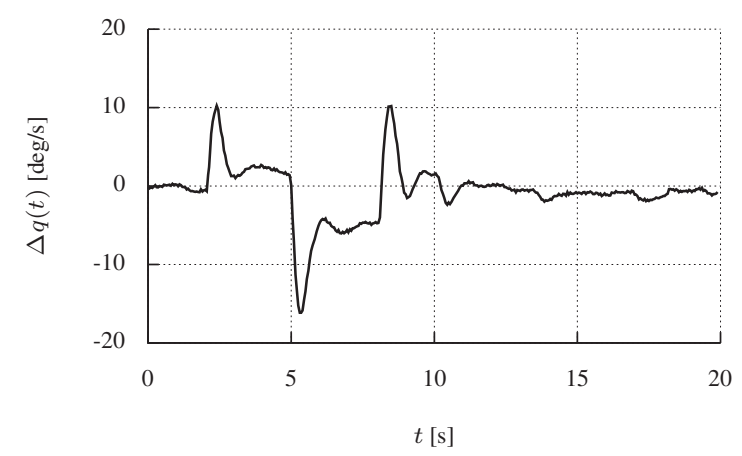

(a) time series data

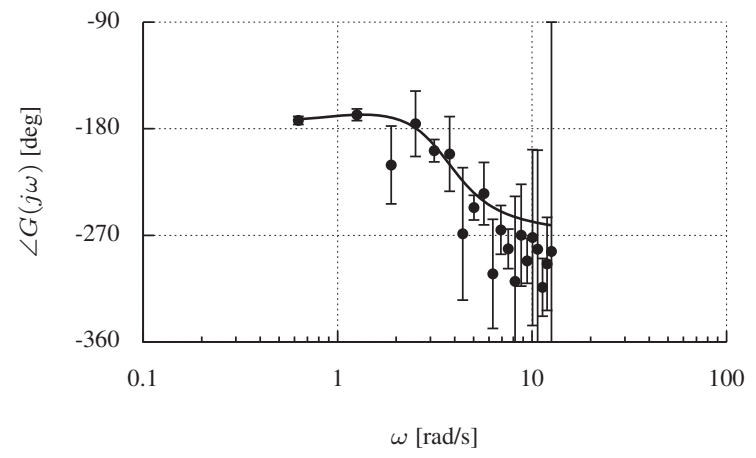

(b) frequency response

Figure 8. Flight test results using a doublet input
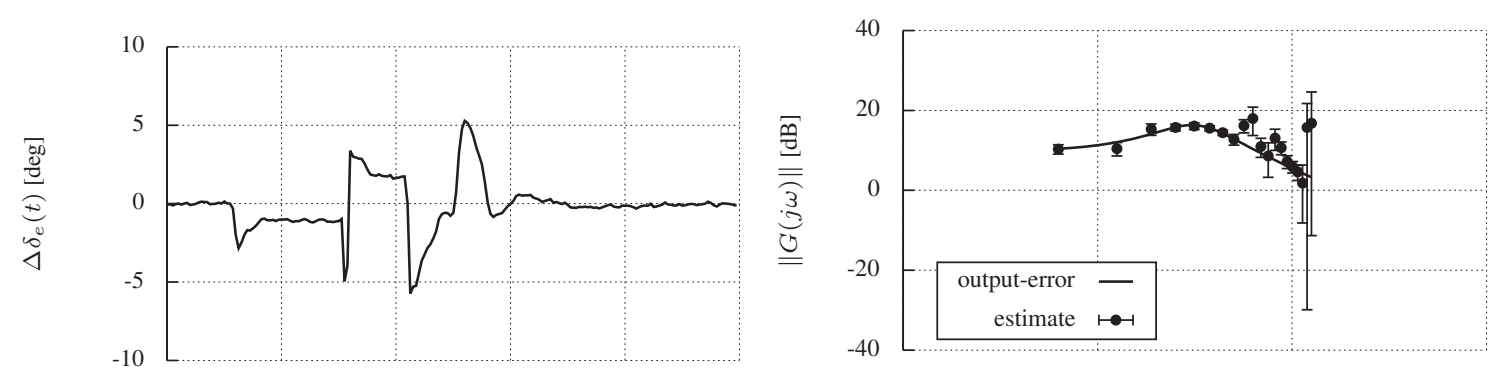

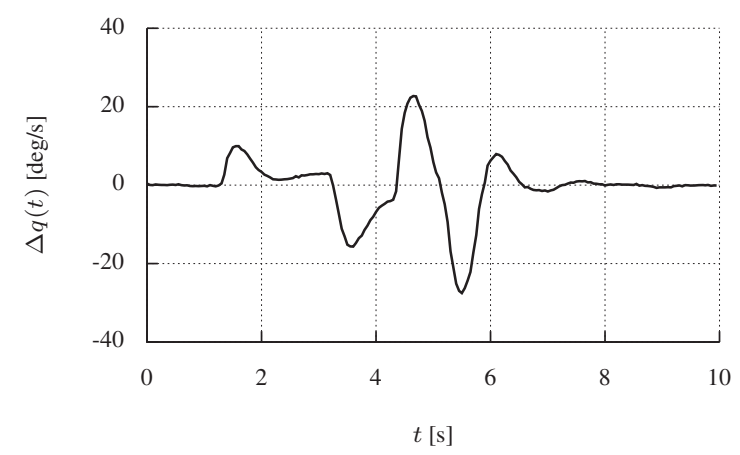

(a) time series data

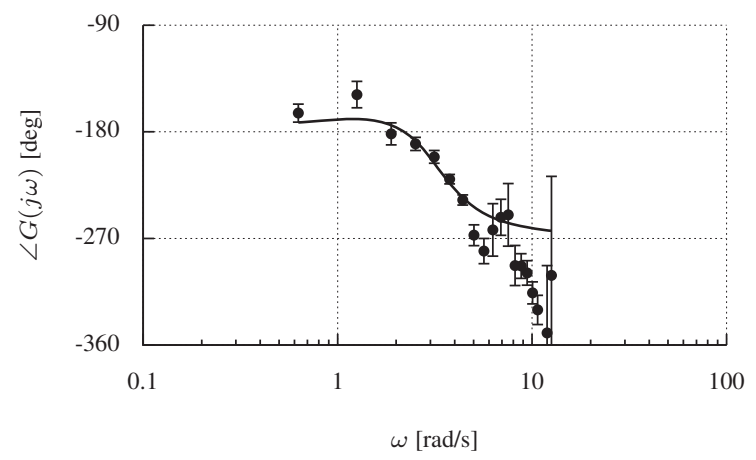

(b) frequency response

Figure 9. Flight test results using a 3-2-1-1 multistep input 

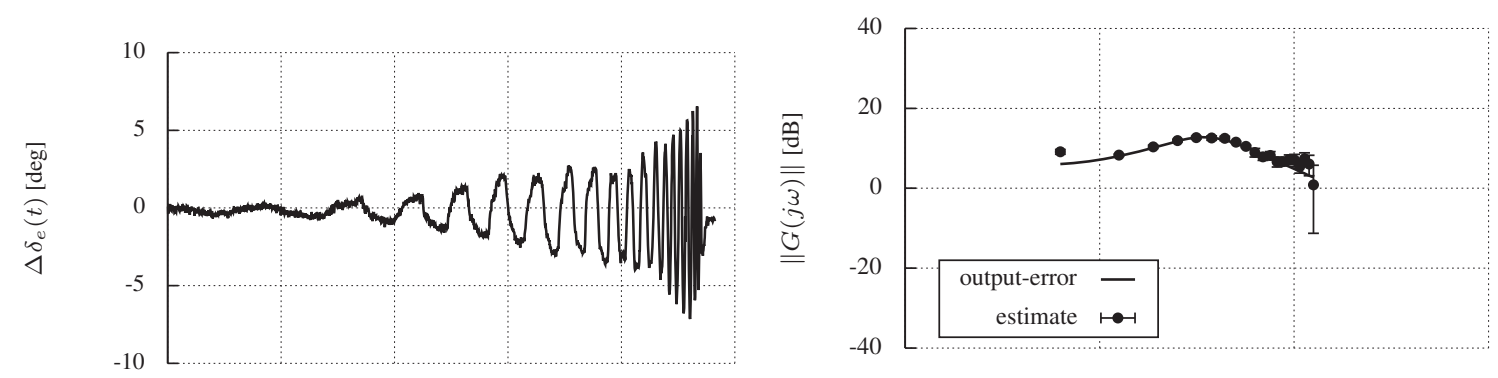

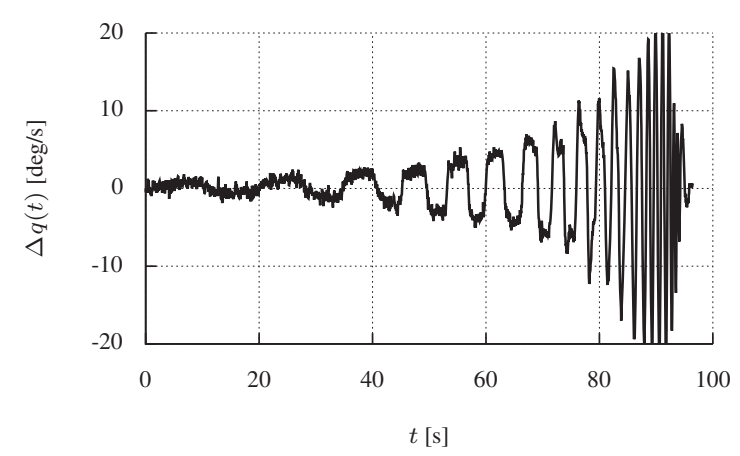

(a) time series data

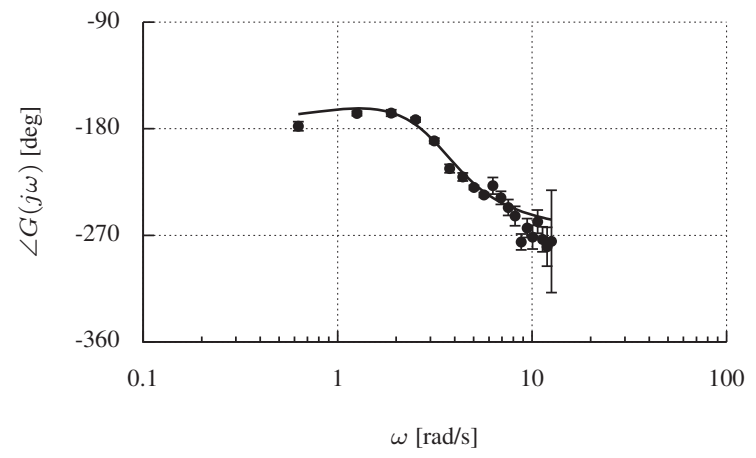

(b) frequency response

Figure 10. Flight test results using a frequency sweep
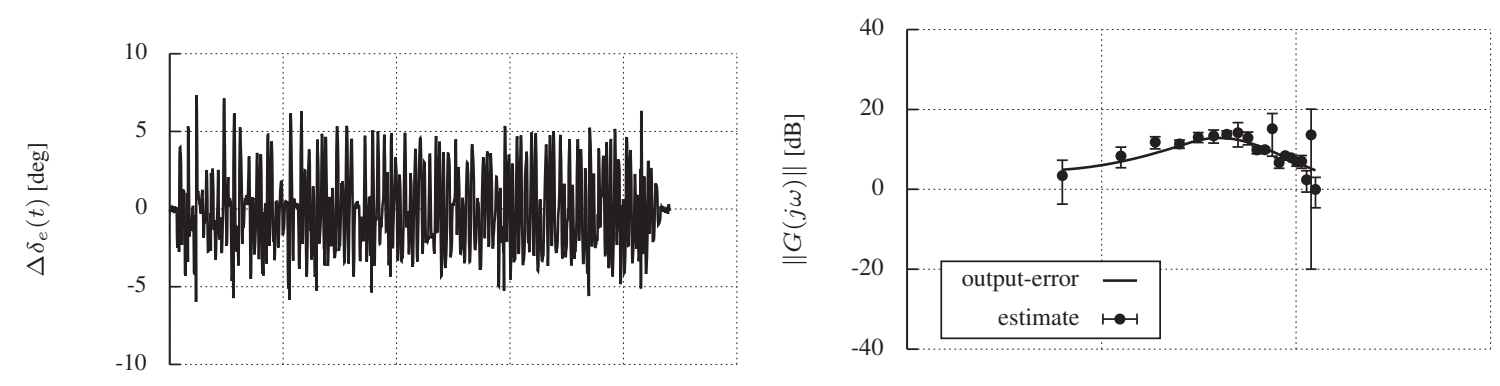

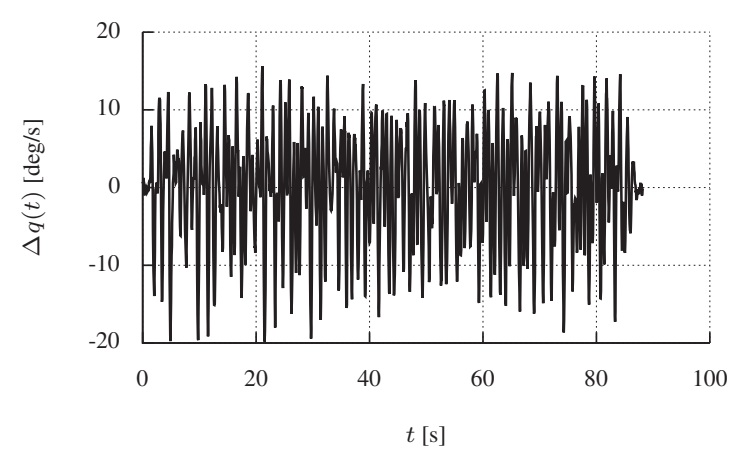

(a) time series data

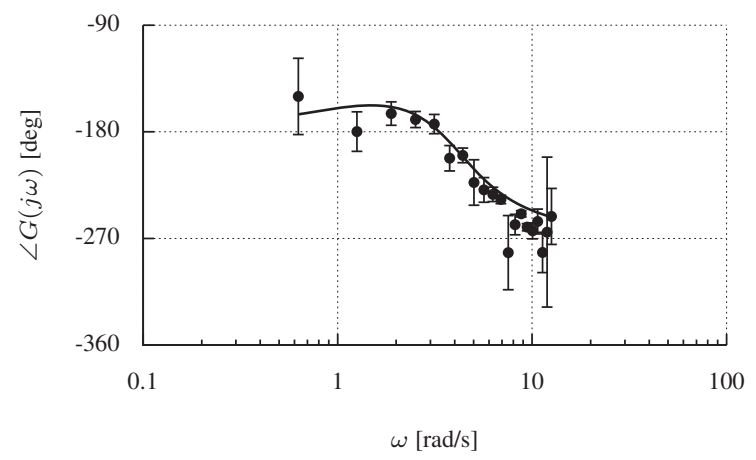

(b) frequency response

Figure 11. Flight test results using a fuzzy input 


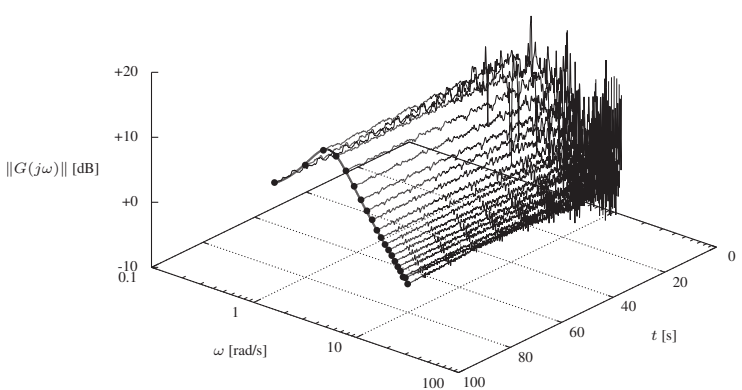

(a) magnitude

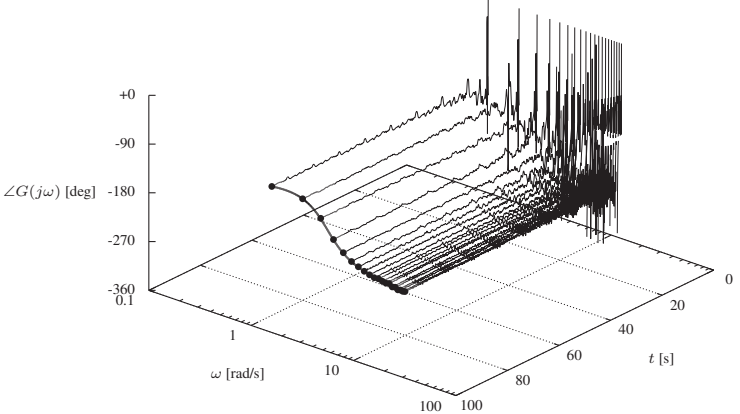

(b) phase angle

Figure 12. Real-time simulation results using a multisine

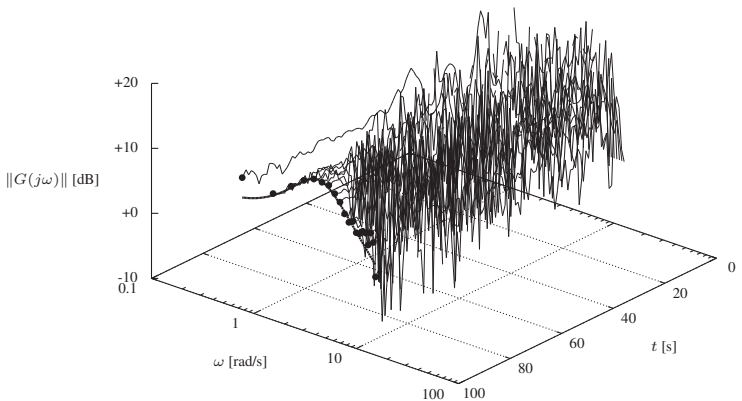

(a) magnitude

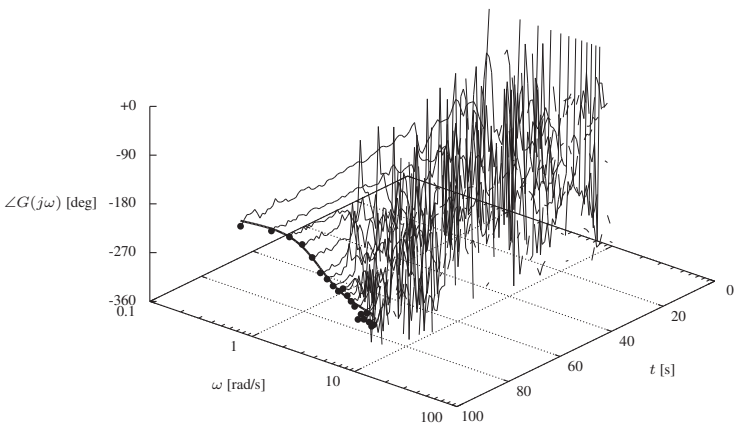

(b) phase angle

Figure 13. Real-time flight test results using a frequency sweep

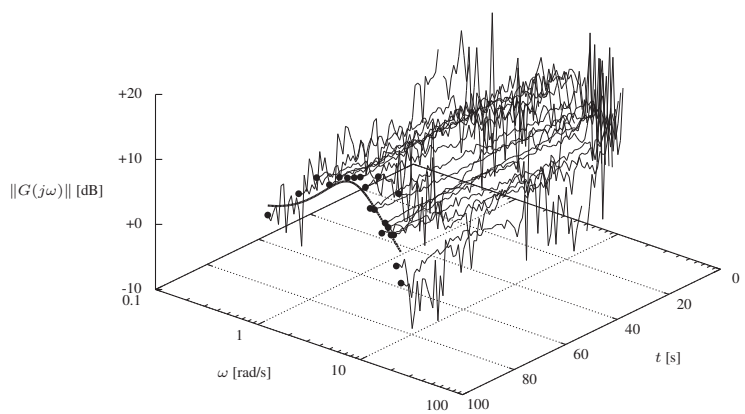

(a) magnitude

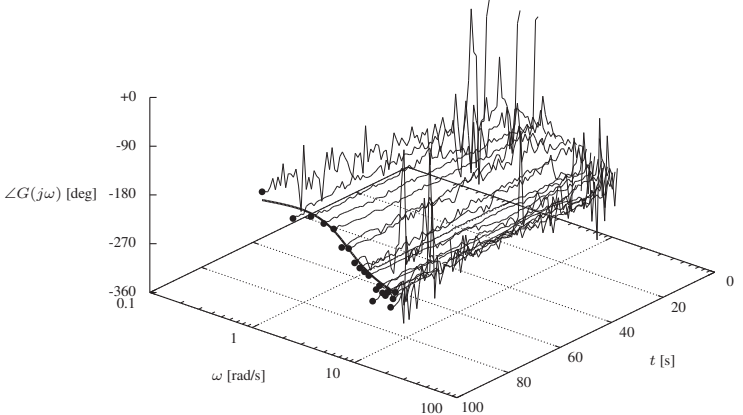

(b) phase angle

Figure 14. Real-time flight test results using a fuzzy input 


\section{Conclusions}

A nonlinear flight dynamics simulation of the F-16 fighter and also a Piper Saratoga general aviation aircraft were used in this study. The F-16 was subjected to multisine, impulse, multistep, and frequency sweep inputs, whereas the Piper Saratoga was subjected to doublet, multistep, frequency sweep, and fuzzy inputs. The findings of this study were:

1. The real-time frequency response estimation method can still be used without multisine inputs, although multisine inputs give superior results in regard to accuracy and efficiency.

2. Piloted inputs should should have sufficient excitation in all frequencies within a specified band width of interest.

3. Applying a new metric called normalized input power $\gamma\left(j \omega_{k}\right) \geq 20 \mathrm{deg}^{2} / \mathrm{s}$ was useful for evaluating which subset of frequency points should be used in the analysis.

4. Evaluating Fourier transforms between $0.1 \mathrm{~Hz}$ and $2.0 \mathrm{~Hz}$, in $0.1 \mathrm{~Hz}$ increments, worked well for estimating the short period mode with most piloted inputs.

In the near future this method will be employed in real time for modeling new aircraft and exotic aircraft configurations with upcoming NASA projects. More research is needed to determine if there is a better method for picking the frequency points for evaluating the Fourier transform, or if there is a method for doing this adaptively as more information becomes available. Although extremely taxing to the pilot, it is possible that if fuzzy inputs can be made mathematically different enough from each other, this method can also be applied for multiple input identification, like it was originally designed.

\section{Acknowledgments}

This work was funded by the NASA Aviation Safety Program and the Vehicle Systems Safety Technologies project. The pilot test inputs were implemented on the Piper Saratoga at the University of Tennessee Space Institute by Borja Martos. Conversations with Eugene Morelli are acknowledged and appreciated. 


\section{References}

${ }^{1}$ Ljung, L., System Identification: Theory for the User, Information and System Sciences Series, Prentice Hill, Upper Saddle River, NJ, 2nd ed., 1999.

${ }^{2}$ Klein, V. and Morelli, E., Aircraft System Identification: Theory and Practice, AIAA Education Series, AIAA, 2006.

${ }^{3}$ Tischler, M. and Remple, R., Aircraft and Rotorcraft System Identification: Engineering Methods with Flight Test Examples, AIAA, 2006.

${ }^{4}$ Bendat, J. and Piersol, A., Random Data: Analysis and Measurement Procedures, John Wiley and Sons, 2nd ed., 1986.

${ }^{5}$ Chen, K. and Shin, J., "Closed Loop Control Law Stability and Performance Analysis Directly Using Open Loop Flight Test Data," No. 2012-4983 in Guidance, Navigation, and Control conference, AIAA, Minneapolis, MN, August 2012.

${ }^{6}$ Balough, D., "Determination of X-36 Stability Margins using Real-Time Frequency Response Techniques," No. 1998-4154, AIAA, 1998.

${ }^{7}$ Sahai, R., Tischler, M., Wei, M., Ng, Y., and Pierce, L., "Flight-Time Identification of Helicopter Slung-Load Frequency Response Characteristics using CIFER," No. 1999-4246, AIAA, 1999.

${ }^{8}$ Field, E., Rossitto, K., and Hodgkinson, J., "Identification of Frequency Responses from Flight Data and their Application for Flying Qualities Analyses," No. 2003-5537, AIAA, 2003.

${ }^{9}$ Lichter, M., Bateman, A., and Balas, G., "Flight Test Evaluation of a Run-Time Stability Margin Estimation Tool," No. 2009-6257, AIAA, 2009.

${ }^{10}$ Zaal, P. and Sweet, B., "Estimation of Time-Varying Pilot Model Parameters," No. 2011-6474, AIAA, 2011.

${ }^{11}$ Baumann, E., "Tailored Excitation for Frequency Response Measurement Applied to the X-43A Flight Vehicle," No. 2006-638, AIAA, 2006.

${ }^{12}$ Morelli, E., "Real-Time Dynamic Modeling: Data Information Requirements and Flight-Test Results," Journal of Aircraft, Vol. 46, No. 6, Nov.-Dec. 2009, pp. 1894-1905.

${ }^{13} \mathrm{Heeg}$, J. and Morelli, E., "Evaluation of Simultaneous Multi-Sine Excitation of the Joined-Wing Sensor Craft Aeroelastic Wind Tunnel Model," No. 2011-1959, AIAA, 2011.

${ }^{14}$ Morelli, E., "Flight Test Maneuvers for Efficient Aerodynamic Modeling," No. 2011-6672 in Atmospheric Flight Mechanics conference, AIAA, Portland, OR, Aug. 2011.

${ }^{15}$ Grauer, J. and Morelli, E., "Real-Time Frequency Response Estimation Using Multi-Sine Inputs and Recursive Fourier Transform," No. 2012-4409 in AIAA Atmospheric Flight Mechanics Conference, AIAA, Minneapolis, MN, August 2012.

${ }^{16}$ Grauer, J. and Morelli, E., "Method for Real-Time Frequency Response and Uncertainty Estimation," Journal of Guidance, Control, and Dynamics, 2013, accessed 10 July 2013. doi: http://arc.aiaa.org/doi/abs/10.2514/1.60795.

${ }^{17}$ Morelli, E., "Multiple Input Design for Real-Time Parameter Estimation in the Frequency Domain," No. REG-360 in 13th Conference on System Identification, IFAC, Rotterdam, Netherlands, Aug. 2003.

${ }^{18}$ Morelli, E., "Flight-Test Experiment Design for Characterizing Stability and Control of Hypersonic Vehicles," Journal of Guidance, Control, and Dynamics, Vol. 32, No. 3, May-June 2009, pp. 949-959.

${ }^{19}$ Morelli, E., "Real-Time Parameter Estimation in the Frequency Domain," Journal of Guidance, Control, and Dynamics, Vol. 23, No. 5, Sept.-Oct. 2000, pp. 812-818.

${ }^{20}$ Nguyen, L., Ogburn, M., Gilbert, W., Kibler, K., Brown, P., and Deal, P., "Simulator Study of Stall/Post-Stall Characteristics of a Fighter Airplane with Relaxed Longitudinal Static Stability," Tech. Rep. TP 1538, NASA, 1979.

${ }^{21}$ Stevens, B. and Lewis, F., Aircraft Control and Simulation, Wiley, 2003.

${ }^{22}$ Koehler, R. and Wilhelm, K., "Auslegung von Eingangssignalen für die Kennwertermittlung," Tech. Rep. IB 154-77/40, Institute für Flugmechanik, Brunswick, Germany, 1977.

${ }^{23}$ Proskawetz, K., "Optimierung stufenförmiger Eingangssignale im Frequenzbereich für die Parameteridentifizierung," Zeirtschrift für Flugwissenshcaften und Weltraumforschung, Vol. 9, No. 6, 1985, pp. 362-370.

${ }^{24}$ Maine, R. and Illif, K., "Application of Parameter Estimation to Aircraft Stability and Control: The Output-Error Approach," Tech. Rep. 1168, NASA, June 1986.

${ }^{25}$ McRuer, D., Ashkenas, I., and Graham, D., Aircraft Dynamics and Automatic Control, Princeton, 1973.

${ }^{26}$ Brandon, J. and Morelli, E., "Nonlinear Aerodynamic Modeling From Flight Data Using Advanced Piloted Maneuvers and Fuzzy Logic," Tech. Rep. TM-2012-217778, NASA, Hampton, VA, October 2012. 